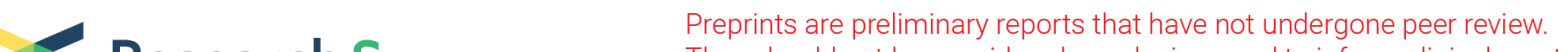 $\begin{array}{ll}\text { Research Square } & \text { They should not be considered conclusive, used to inform clinical practice, } \\ \text { or referenced by the media as validated information. }\end{array}$
}

\section{Lung Stereotactic Radiation Therapy: Intercomparison of Several Irradiation Devices in Terms of Outcome and Predictive Factors}

\section{Eymeric Le Reun}

CHUV: Centre Hospitalier Universitaire Vaudois

\section{Alessio Casutt}

CHUV: Centre Hospitalier Universitaire Vaudois

\section{André Durham}

Geneve University Hospitals: Hopitaux Universitaires Geneve

\section{Hasna Bouchaab}

CHUV: Centre Hospitalier Universitaire Vaudois

\section{Edouard Romano}

CHUV: Centre Hospitalier Universitaire Vaudois

\section{Alban Lovis}

CHUV: Centre Hospitalier Universitaire Vaudois

\section{Thorsten Krueger}

CHUV: Centre Hospitalier Universitaire Vaudois

\section{Christophe Von Garnier}

CHUV: Centre Hospitalier Universitaire Vaudois

\section{Esat-Mahmut Ozsahin}

CHUV: Centre Hospitalier Universitaire Vaudois

Rémy Kinj ( $\Delta$ remykinj@gmail.com )

Centre Hospitalier Universitaire Vaudois Departement d'oncologie CHUV-UNIL https://orcid.org/0000-00019283-7227

\section{Research Article}

Keywords: CyberKnife, Lung tumor, Metastasis, NSCLC, Predictive factors, SBRT, Stereotactic radiotherapy, dosimetric comparison

Posted Date: January 6th, 2022

DOl: https://doi.org/10.21203/rs.3.rs-1213819/v1

License: (9) (i) This work is licensed under a Creative Commons Attribution 4.0 International License. Read Full License 


\section{Abstract}

Background: Stereotactic body radiotherapy (SBRT) is recommended for the treatment of inoperable early stage non-small-cell lung cancer and lung oligometastases. The radiation oncology department of the Lausanne University Hospital (CHUV) gathers three different radiotherapy devices able to treat pulmonary lesions in SBRT conditions: CyberKnife ${ }^{\circledR}(\mathrm{CK})$, Helical Tomotherapy ${ }^{\circledR}(\mathrm{HT})$, and volumetric modulated arc therapy (VMAT). The aim of this study is to define the patients' outcome in terms of irradiation efficacy and toxicities after lung SBRT depending of the choice of the SBRT technique.

Methods: We retrospectively analyzed the clinical, radiological, and dosimetric data of patients with primary lung tumor or pulmonary oligometastases treated with SBRT between January 2016 and February 2020. We analyzed descriptive data using the Chi-2 test for proportions and the T-test for means comparisons, survival data by the Kaplan-Meier method and comparisons between groups by the Log-rank test and Cox-regression.

Results: We identified 111 patients mostly in good condition (82.9\% PS 0-1) with a median age of 71.4 years. One hundred forty-two lesions were treated with a typical fractionation of $55 \mathrm{~Gy}$ in 5 fractions, delivered by CK (59.9\%), VMAT (38.0\%), or HT (2.1\%). Compared to other techniques, CK technique allowed to treat comparable gross tumor volume (GTV; 2.1 vs $1.4 \mathrm{cc}, \mathrm{p}=0.84$ ) with smaller planning treatment volume (PTV; $12.3 \mathrm{vs} 21.9 \mathrm{cc}, \mathrm{p}=$ 0.013), and was associated with a lower mean lung dose (MLD; 2.6 vs $4.1 \mathrm{~Gy}, \mathrm{p}<0.001$ ), a lower V5 (13.5 vs 19.9 $c c, p=0.002)$ and a lower V20 (2.3 vs $5.4 \mathrm{cc}, \mathrm{p}<0.001)$. Local control rates at 2 years were not different depending on the irradiation device, respectively of $96.2 \%$ (range, 90.8-100) and 98.1\% (range, 94.4-100), $p=0.68$. Toxicity incidence was significantly increased with V5 value $>17.2 \%(56.0$ vs $77.4 \%, p=0.021)$.

Conclusions: Compared to other SBRT techniques, CK treatments permitted to treat comparable GTV with reduced PTV, MLD, V20, and V5. The dosimetric benefit of CK SBRT was not associated with a clear clinical benefit, with comparable outcome in terms of control rates and toxicity. Toxicity incidence was less frequent when reducing the V5. The use of CK is particularly attractive in case of multiple courses of lung SBRT or in case of local relapse requiring lung re-irradiation.

Trial registration: Registered on February 24th 2021, ID 2021-00267, with the authorization of the CER-VD ethics committee (Switzerland).

\section{Background}

Lung cancer is the most common cause of cancer-related death(1). The introduction of pulmonary stereotactic body radiotherapy (SBRT) for early malignancies has been correlated to a reduction in the number of patients who remain untreated(2,3). SBRT is recommended for early stage non-small-cell lung cancer (NSCLC), either in medically or surgically inoperable patients, or in well-selected operable patients, according to clinical guidelines(4-7). In addition, various SBRT indications for lung oligometastases are well known(8).

The radiation oncology department of the Lausanne University Hospital (CHUV) gathers three different radiotherapy devices able to treat pulmonary lesions in SBRT conditions: Synergy ${ }^{\circledR}$ (Elekta, Sweden), Helical Tomotherapy ${ }^{\circledR}$ (Accuray, USA), and CyberKnife ${ }^{\circledR}$ (Accuray, USA).

Synergy ${ }^{\circledR}$ is a linear accelerator (linac) that uses volumetric modulated arc treatment (VMAT). The second device, termed helical Tomotherapy ${ }^{\circledR}(\mathrm{HT})$, combines a megavoltage computed tomography (MV-CT) and a miniaturized 
linac to provide image-guided radiation therapy. During helicolidal irradiation, the machine slowly spins around the patient while the table travels longitudinally. When using Synergy ${ }^{\circledR}$ and $H T$, an internal target volume is either determined, on a 4D CT or in obstructed inspiration owing to a respiratory servo system called Active Breath Control $\circledast(A B C)$. When using a 4D-CT for planning, the gross tumor volume (GTV) of each phase is combined into an internal target volume (ITV). This method is the most commonly used when irradiation is done using VMAT or HT. The planning target volume (PTV) corresponds to an ITV with a 3-mm margin in this configuration. The CyberKnife ${ }^{\circledR}(\mathrm{CK})$ is a linear accelerator with a head and a robotic arm, totally dedicated to SBRT. Several tracking systems allow for alternative irradiation treatments with this device: i) Synchrony ${ }^{\circledR}$ : respiratory monitoring with placement through fiducial markers; ii) X-sight Spine®: monitoring of the spine associated with a ITV delineated based on a 4D CT-scanner; and iii) X-sight Lung ${ }^{\circledR}$ with monitoring of the tumor itself $(9,10)$.

When using the CK tracking method, the GTV is immediately expanded by $3 \mathrm{~mm}$ to the PTV. As a result, CK may be able to lower GTV to PTV margins while avoiding ITV-related volume expansion.

Within the same radiation oncology department, only a few institutions have different devices able to perform pulmonary SBRT. The aim of this study is to determine the patients' outcomes in terms of irradiation efficacy and toxicities after lung SBRT according to the different SBRT techniques.

\section{Methods}

Between January 2016 and February 2020, we reviewed the clinical, radiological, and dosimetric data of consenting patients with primary lung tumors or pulmonary oligometastases treated by SBRT at CHUV. All patients were selected for SBRT after a multidisciplinary thoracic oncological tumor board discussion, and signed an informed consent form for irradiation. Patients having a lung tumor treated with a method other than SBRT, such as surgery or conventional radiotherapy, were not included.

Clinical data such as the patient's age, sex, WHO performance index, smoking status, comorbidities, previous medical history including previous irradiation or thoracic surgery, cancer histology, pulmonary location of the tumor (affected lobe, central or peripheral), and the reason for radio-oncological treatment rather than the surgical one, were recorded. The reason for choosing SBRT modality was at the discretion of the physician.

The fractionation schedule, GTV, PTV, PTV dose, dose received by the lungs, i.e. the mean lung dose (MLD), the percent of the volume of the lung receiving $5 \mathrm{~Gy}$ (V5) and $20 \mathrm{~Gy}$ (V20), and composite dosimetry in the event of multiple courses of irradiation were all part of the dosimetric data record.

Radiological data included tumor response after SBRT (stable disease, complete or partial response to treatment), and any recurrence (local, locoregional or distant metastatic) were described.

Clinical and radiological data were extracted from the institutional clinical software Soarian $\circledast$ (Cerner, United States) and Archimède ${ }^{\circledR}$ (CHUV) software; dosimetric data were recorded from RayStation ${ }^{\circledR}$ (RaySearch Laboratories, Sweden) and VelocityTM (Varian Medical Systems, USA) radiotherapy software. Data were then anonymized by assigning to each patient a neutral identifier of 4 digits, and the study was registered on February 24th 2021, ID 2021-00267, with the authorization of the CER-VD ethics committee (Switzerland). Statistical analyses were performed using the JMP® 15 software. We analyzed descriptive data using the Chi-2 test for proportions and the T-test for mean comparisons. Survival data were analyzed by the Kaplan-Meier method, and 
comparisons between groups by the Log-rank test. Confidence intervals $(\mathrm{Cl})$ were computed from standard errors. If $p$-value was $\leq 0.15$ in univariate analysis, the variable was tested in multivariate analysis through Coxregression test.

\section{Results}

\section{Patients' and treatment characteristics}

We identified 111 patients, mostly smoker (64.9\%) men (65.8\%) in good condition (82.9\% PS 0-1) with a median age of 71.4 years. Patients presenting primary pulmonary tumors $(n=57)$ and lung metastases $(n=54)$ were found in equal numbers. Patients, who were medically inoperable due to comorbidities, were the most common cause for not selecting for surgery, and instead choosing SBRT (44.2\%). We treated 142 pulmonary lesions, the majority being peripheral tumors $(73.9 \%)$. More than half of the patients had a history of thoracic surgery (52.3\%), and about a quarter had a history of thoracic irradiation (23.4\%). The prescribed dose ranged from 34-60 Gy over 1-8 fractions with a typical fractionation of $55 \mathrm{~Gy}$ in 5 fractions (66.7\%), delivered by CK (59.9\%), VMAT (38.0\%), or HT (2.1\%). A large majority of patients benefited from a tumor tracking through fiducials (51.4\%), and many of others from an ITV Planification with 4D-CT (33.8\%). The median MLD was $3.7 \mathrm{~Gy}$, with a median V5 at $17.2 \%$. In case of iterative irradiation, the median composite MLD and composite V 5 reached 7.9 Gy and $37.9 \%$ respectively. Other patients and treatment characteristics are presented in Table 1. 
Table 1

Descriptive statistics for patient's demographics, treatment and outcome

\begin{tabular}{|c|c|c|c|}
\hline Patients & $\mathrm{n}=1$ & & \\
\hline Age: median - minimum - maximum [years] & 71.4 & 32.5 & 91.6 \\
\hline Gender & \multicolumn{3}{|c|}{$n=111$} \\
\hline Male & \multicolumn{3}{|c|}{$73(65.8 \%)$} \\
\hline Female & \multicolumn{3}{|c|}{$38(34.2 \%)$} \\
\hline WHO performance status & \multicolumn{3}{|c|}{$\mathrm{n}=111$} \\
\hline 0 & \multicolumn{3}{|c|}{$34(30.6 \%)$} \\
\hline 1 & \multicolumn{3}{|c|}{$58(52.3 \%)$} \\
\hline 2 & \multicolumn{3}{|c|}{$17(15.3 \%)$} \\
\hline 3 & \multicolumn{3}{|c|}{$1(<1 \%)$} \\
\hline 4 & \multicolumn{3}{|c|}{$1(<1 \%)$} \\
\hline Smoker & \multicolumn{3}{|c|}{$\mathrm{n}=111$} \\
\hline Yes & \multicolumn{3}{|c|}{$72(64.9 \%)$} \\
\hline No & \multicolumn{3}{|c|}{$39(35.1 \%)$} \\
\hline History of cancer & \multicolumn{3}{|c|}{$\mathrm{n}=111$} \\
\hline Yes & \multicolumn{3}{|c|}{$43(38.7 \%)$} \\
\hline No & \multicolumn{3}{|c|}{$68(61.3 \%)$} \\
\hline History of thoracic irradiation & \multicolumn{3}{|c|}{$\mathrm{n}=111$} \\
\hline Yes & \multicolumn{3}{|c|}{$26(23.4 \%)$} \\
\hline - one previous irradiation & \multicolumn{3}{|c|}{$23(88.5 \%)$} \\
\hline - two previous irradiations & \multicolumn{3}{|c|}{$3(11.5 \%)$} \\
\hline No & \multicolumn{3}{|c|}{$85(76.6 \%)$} \\
\hline $1^{\text {st }}$ history: & \multicolumn{3}{|c|}{$n=26$} \\
\hline - Total dose: median - minimum - maximum [Gy] & 60 & 8 & 70 \\
\hline - Dose/fraction: median - minimum - maximum [Gy] & 2 & 1.8 & 30 \\
\hline $2^{\text {nd }}$ history: & \multicolumn{3}{|c|}{$\mathrm{n}=3$} \\
\hline - Total dose: median - minimum - maximum [Gy] & 36 & 25 & 60 \\
\hline - Dose/fraction: median - minimum - maximum [Gy] & 6 & 5 & 12 \\
\hline History of thoracic surgery & \multicolumn{3}{|c|}{$\mathrm{n}=111$} \\
\hline
\end{tabular}




\begin{tabular}{|c|c|}
\hline Yes: & $58(52.3 \%)$ \\
\hline - wedge resection only & $13(22.4 \%)$ \\
\hline - segmentectomy & $5(8.6 \%)$ \\
\hline - lobectomy & $28(48.3 \%)$ \\
\hline - bilobectomy & $6(10.3 \%)$ \\
\hline - pneumonectomy & $4(6.9 \%)$ \\
\hline - others & $2(3.5 \%)$ \\
\hline No & $53(47.7 \%)$ \\
\hline Reason for non-operability: & $\mathrm{n}=111$ \\
\hline - surgery refused by patient & $5(4.5 \%)$ \\
\hline - surgically non operable & $24(21.6 \%)$ \\
\hline - medically non operable & $49(44.2 \%)$ \\
\hline - systemicdisease & $33(29.7 \%)$ \\
\hline Primary or metastasis & $\mathrm{n}=111$ \\
\hline Primary tumor & $57(51.4 \%)$ \\
\hline Metastasis: & $54(48.6 \%)$ \\
\hline - lung & $22(40.7 \%)$ \\
\hline - digestive & $14(25.9 \%)$ \\
\hline - ENT & $7(13.0 \%)$ \\
\hline - melanoma & $5(9.3 \%)$ \\
\hline - pleura & $1(1.8 \%)$ \\
\hline - others & $5(9.3 \%)$ \\
\hline Tumor localization: & $n=142$ \\
\hline Left lung: & $64(45.1 \%)$ \\
\hline - upper left lobe & $32(50.0 \%)$ \\
\hline - lower left lobe & $32(50.0 \%)$ \\
\hline Right lung: & $74(52.1 \%)$ \\
\hline - upper lobe & $35(47.3 \%)$ \\
\hline - middle lobe & $6(8.1 \%)$ \\
\hline - lower lobe & $29(39.2 \%)$ \\
\hline - two lobes & $4(5.4 \%)$ \\
\hline
\end{tabular}




\begin{tabular}{|c|c|}
\hline Bilateral & $4(2.8 \%)$ \\
\hline Central & $32(22.6 \%)$ \\
\hline Peripheral & 105 (73.9\%) \\
\hline Central + peripheral & $5(3.5 \%)$ \\
\hline \multicolumn{2}{|l|}{ Histology: } \\
\hline Histological proof: & $\mathrm{n}=111$ \\
\hline - Yes & $77(69.4 \%)$ \\
\hline - No & $34(30.6 \%)$ \\
\hline Histological type: & $\mathrm{n}=111$ \\
\hline - NSCLC & $3(2.7 \%)$ \\
\hline - adenocarcinoma & $43(38.7 \%)$ \\
\hline - squamouscell & $30(27.1 \%)$ \\
\hline - adenoidcystic carcinoma & $3(2.7 \%)$ \\
\hline - sarcoma & $2(1.8 \%)$ \\
\hline - melanoma & $5(4.5 \%)$ \\
\hline - hepatocellularcarcinoma & $1(0.9 \%)$ \\
\hline - others & $24(21.6 \%)$ \\
\hline TNM at diagnosis & $\mathrm{n}=111$ \\
\hline \multicolumn{2}{|l|}{$\mathrm{T}:$} \\
\hline$-\mathrm{TX}$ & $9(8.1 \%)$ \\
\hline - Tis & $1(<1 \%)$ \\
\hline$-\mathrm{T} 1$ & $51(45.9 \%)$ \\
\hline$-\mathrm{T} 2$ & $28(25.2 \%)$ \\
\hline$-\mathrm{T3}$ & $11(9.9 \%)$ \\
\hline$-\mathrm{T} 4$ & $11(9.9 \%)$ \\
\hline $\mathrm{N}:$ & $\mathrm{n}=111$ \\
\hline$-N X$ & $8(7.2 \%)$ \\
\hline$-\mathrm{NO}$ & $75(67.6 \%)$ \\
\hline$-\mathrm{N} 1$ & $13(11.7 \%)$ \\
\hline$-\mathrm{N} 2$ & $11(9.9 \%)$ \\
\hline
\end{tabular}




\begin{tabular}{|c|c|c|c|}
\hline$-N 3$ & \multicolumn{3}{|c|}{$4(3.6 \%)$} \\
\hline M: & \multicolumn{3}{|c|}{$\mathrm{n}=111$} \\
\hline$-M X$ & \multicolumn{3}{|c|}{$8(7.2 \%)$} \\
\hline$-\mathrm{MO}$ & \multicolumn{3}{|c|}{$75(67.6 \%)$} \\
\hline$-M 1$ & \multicolumn{3}{|c|}{$28(25.2 \%)$} \\
\hline Number of metastatic sites: & \multicolumn{3}{|c|}{$\mathrm{n}=111$} \\
\hline-1 & \multicolumn{3}{|c|}{$31(27.9 \%)$} \\
\hline-2 & \multicolumn{3}{|c|}{$21(18.9 \%)$} \\
\hline$-\geqslant 3$ & \multicolumn{3}{|c|}{$2(1.8 \%)$} \\
\hline Treatments & \multicolumn{3}{|c|}{$n=142$} \\
\hline PTV volume: & \multicolumn{3}{|c|}{$n=133$} \\
\hline median - minimum - maximum [cc] & 8.1 & 1.3 & 190.4 \\
\hline Radiotherapy technique: & \multicolumn{3}{|c|}{$n=142$} \\
\hline - CyberKnife® & \multicolumn{3}{|c|}{$85(59.9 \%)$} \\
\hline - VMAT & \multicolumn{3}{|c|}{$54(38.0 \%)$} \\
\hline - Tomotherapy® & \multicolumn{3}{|c|}{$3(2.1 \%)$} \\
\hline SBRT modality: & \multicolumn{3}{|c|}{$n=142$} \\
\hline$-A B C$ & \multicolumn{3}{|c|}{$5(3.5 \%)$} \\
\hline - fiducials & \multicolumn{3}{|c|}{$73(51.4 \%)$} \\
\hline - ITV & \multicolumn{3}{|c|}{$48(33.8 \%)$} \\
\hline - Mid-ventilation & \multicolumn{3}{|c|}{$2(1.4 \%)$} \\
\hline - X-sight lung $®$ & \multicolumn{3}{|c|}{$3(2.1 \%)$} \\
\hline - X-sightspine $®$ & \multicolumn{3}{|c|}{$11(7.8 \%)$} \\
\hline Dosimetry: & \multicolumn{3}{|c|}{$n=142$} \\
\hline Total dose: median - minimum - maximum [Gy] & 55 & 34 & 60 \\
\hline Duration: median - minimum - maximum [days] & 11 & 1 & 25 \\
\hline Number of fractions: median - minimum - maximum & 5 & 1 & 8 \\
\hline Current treatment: & \multicolumn{3}{|c|}{$n=130$} \\
\hline - Mean lung dose (MLD): median - minimum - maximum [Gy] & 3.7 & 0.2 & 22.3 \\
\hline - Volume of lung receiving 5Gy (V5\%): median - minimum - maximum [\%] & 17.2 & 2.1 & 93.6 \\
\hline
\end{tabular}




\begin{tabular}{|c|c|c|c|}
\hline \multirow{2}{*}{$\begin{array}{l}\text { Cumulative dosimetry: } \\
\text { - MLD composite: median - minimum - maximum [Gy] }\end{array}$} & \multicolumn{3}{|c|}{$n=38$} \\
\hline & 7.9 & 0.9 & 22.3 \\
\hline - V5\% composite: median - minimum - maximum [\%] & 37.9 & 4.29 & 93.6 \\
\hline \multicolumn{4}{|l|}{ Outcome } \\
\hline Toxicity: & \multicolumn{3}{|c|}{$n=111$} \\
\hline Yes & \multicolumn{3}{|c|}{$76(68.5 \%)$} \\
\hline No & \multicolumn{3}{|c|}{$35(31.5 \%)$} \\
\hline Acute toxicity: & \multicolumn{3}{|c|}{$\mathrm{n}=111$} \\
\hline Yes & \multicolumn{3}{|c|}{$63(56.8 \%)$} \\
\hline - grade 1 & \multicolumn{3}{|c|}{$50(79.3 \%)$} \\
\hline - grade 2 & \multicolumn{3}{|c|}{$11(17.5 \%)$} \\
\hline - grade 3 & \multicolumn{3}{|c|}{$2(3.2 \%)$} \\
\hline No & \multicolumn{3}{|c|}{$48(43.2 \%)$} \\
\hline Late toxicity: & \multicolumn{3}{|c|}{$\mathrm{n}=111$} \\
\hline Yes & \multicolumn{3}{|c|}{$29(26.1 \%)$} \\
\hline - grade 1 & \multicolumn{3}{|c|}{$13(44.8 \%)$} \\
\hline - grade 2 & \multicolumn{3}{|c|}{$13(44.8 \%)$} \\
\hline - grade 3 & \multicolumn{3}{|c|}{$3(10.4 \%)$} \\
\hline No & \multicolumn{3}{|c|}{$82(73.9 \%)$} \\
\hline Best local response: & \multicolumn{3}{|c|}{$n=142$} \\
\hline - not known & \multicolumn{3}{|c|}{$4(2.8 \%)$} \\
\hline - tumor progression & \multicolumn{3}{|c|}{$1(0.7 \%)$} \\
\hline - complete response & \multicolumn{3}{|c|}{$13(9.2 \%)$} \\
\hline - partial response & \multicolumn{3}{|c|}{$48(33.8 \%)$} \\
\hline - stable disease & \multicolumn{3}{|c|}{$76(53.5 \%)$} \\
\hline Recurrence: & \multicolumn{3}{|c|}{$\mathrm{n}=111$} \\
\hline Yes & \multicolumn{3}{|c|}{$42(37.8 \%)$} \\
\hline - local recurrence & \multicolumn{3}{|c|}{$3(7.1 \%)$} \\
\hline - regional recurrence & \multicolumn{3}{|c|}{$5(11.9 \%)$} \\
\hline
\end{tabular}




\begin{tabular}{|l|l|} 
- distant metastasis & $34(81.0 \%)$ \\
Second primary & $69(62.2 \%)$ \\
\hline Survival: & $10(9.0 \%)$ \\
Alive & $\mathrm{n}=111$ \\
- without disease & $78(70.3 \%)$ \\
- with disease & $46(59.0 \%)$ \\
Dead & $32(41.0 \%)$ \\
- without disease & $33(29.7 \%)$ \\
- with disease & $12(36.4 \%)$ \\
& $21(63.6 \%)$ \\
\hline
\end{tabular}

MLD : Mean Lung Dose; V5\% : percentage of lung receiving 5Gy

\section{Local control}

Local response could be achieved in 138 out of 142 lesions, while local control (LC) was $96.8 \%$ (92.3-100\%, Cl $95 \%)$ at 2 years and $94.8 \%(90.3-100 \%, C I$ 95\%) at 3 years. LC at 2 and 3 years significantly decreased with age, from $100-93.4 \%$ and $88.9 \%$, respectively when patients were older than 71 years. $(p=0.035)$, as shown in Table 2. LC was not influenced by the other treatment characteristics: the choice of radiotherapy device, neither by the SBRT modality, nor by the dose/fractionation scheme. We observed 2 mediastinal relapse, corresponding to a loco-regional control rate of $96.2 \%$ at 1 year and $94.4 \%$ at 2 years, respectively. 
Table 2

Predictive factors for local control

\begin{tabular}{|c|c|c|c|c|c|}
\hline Variable & $\begin{array}{l}2-y \text { local control } \\
(\%)\end{array}$ & $95 \% \mathrm{Cl}$ & $\begin{array}{l}\text { 3-y local control } \\
\text { (\%) }\end{array}$ & $95 \% \mathrm{Cl}$ & $p$-value \\
\hline All $(n=138)$ & 96.8 & $\begin{array}{l}92.3- \\
100\end{array}$ & 94.8 & $\begin{array}{l}90.3- \\
100\end{array}$ & \\
\hline $\begin{array}{l}\text { Age [years]: } \\
\begin{array}{l}\leqslant 71(n=66) \\
>71(n=72)\end{array}\end{array}$ & $\begin{array}{l}100 \\
93.4\end{array}$ & $\begin{array}{l}85.2- \\
100\end{array}$ & $\begin{array}{l}100 \\
88.9\end{array}$ & $\begin{array}{l}77.6- \\
100\end{array}$ & $0.035^{*}$ \\
\hline $\begin{array}{l}\text { Gender: } \\
\text { Women }(n=45) \\
\text { Men }(n=93)\end{array}$ & $\begin{array}{l}97.8 \\
96.1\end{array}$ & $\begin{array}{l}93.3- \\
100 \\
90.5- \\
100\end{array}$ & $\begin{array}{l}90.8 \\
96.1\end{array}$ & $\begin{array}{l}70.1- \\
100 \\
90.5- \\
100\end{array}$ & 0.466 \\
\hline Radiotherapy technique: & 96.2 & $\begin{array}{l}90.8- \\
100 \\
94.4- \\
100\end{array}$ & 93.3 & $\begin{array}{l}85.6- \\
100 \\
94.4- \\
100\end{array}$ & 0.893 \\
\hline CyberKnife $(n=82)$ & 98.1 & $\begin{array}{l}44.9- \\
100\end{array}$ & 98.1 & $\begin{array}{l}44.9- \\
100\end{array}$ & 0.216 \\
\hline VMAT $(n=53)$ & 100 & $\begin{array}{l}88.6^{-} \\
100\end{array}$ & 100 & $\begin{array}{l}82.5- \\
100\end{array}$ & \\
\hline Tomotherapy $(n=3)$ & 80 & & 80 & & \\
\hline SBRT modality: & 95.3 & & 91.8 & & \\
\hline$A B C(n=4)$ & 100 & & 100 & & \\
\hline Fiducials $(n=70)$ & 100 & & 100 & & \\
\hline ITV $(n=48)$ & 100 & & 100 & & \\
\hline $\begin{array}{l}\text { Mid-ventilation }(n=2) \\
\text { X-sight lung }(n=3) \\
\text { X-sight spine }(n=11)\end{array}$ & 100 & & 100 & & \\
\hline Dose [Gy]: & 100 & $\begin{array}{l}90.8^{-} \\
100\end{array}$ & 100 & $\begin{array}{l}44.9- \\
100\end{array}$ & 0.990 \\
\hline $\begin{array}{l}\leqslant 50(n=10) \\
54(n=15)\end{array}$ & $\begin{array}{l}100 \\
96.2\end{array}$ & $\begin{array}{l}86.8^{-} \\
100\end{array}$ & $\begin{array}{l}80 \\
96.2\end{array}$ & $\begin{array}{l}90.8^{-} \\
100\end{array}$ & \\
\hline $\begin{array}{l}55(n=92) \\
60(n=21)\end{array}$ & 95.5 & & 95.5 & $\begin{array}{l}86.8- \\
100\end{array}$ & \\
\hline
\end{tabular}

Missing values $(n=4)$ 


\begin{tabular}{|c|c|c|c|c|c|}
\hline Variable & $\begin{array}{l}2-y \text { local control } \\
(\%)\end{array}$ & $95 \% \mathrm{Cl}$ & $\begin{array}{l}3-y \text { local control } \\
(\%)\end{array}$ & $95 \% \mathrm{Cl}$ & $p$-value \\
\hline $\begin{array}{l}\text { Treatment duration [days]: } \\
\leqslant 11(n=48) \\
>11(n=90)\end{array}$ & $\begin{array}{l}100 \\
94.9\end{array}$ & $\begin{array}{l}88.8- \\
100\end{array}$ & $\begin{array}{l}95.7 \\
94.9\end{array}$ & $\begin{array}{l}87.4- \\
100 \\
88.8- \\
100\end{array}$ & 0.553 \\
\hline $\begin{array}{l}\text { History of cancer: } \\
\text { No }(n=80) \\
\text { Yes }(n=58)\end{array}$ & $\begin{array}{l}97.4 \\
96.2\end{array}$ & $\begin{array}{l}93.9- \\
100 \\
88.8- \\
100\end{array}$ & $\begin{array}{l}93.8 \\
96.2\end{array}$ & $\begin{array}{l}85.7- \\
100 \\
88.8- \\
100\end{array}$ & 0.519 \\
\hline $\begin{array}{l}\text { History of thoracic irradiation: } \\
\text { No }(n=90) \\
\text { Yes }(n=48)\end{array}$ & $\begin{array}{l}96.4 \\
97.9\end{array}$ & $\begin{array}{l}91.3- \\
100 \\
93.9- \\
100\end{array}$ & $\begin{array}{l}93.4 \\
97.9\end{array}$ & $\begin{array}{l}85.9- \\
100 \\
93.9- \\
100\end{array}$ & 0.758 \\
\hline $\begin{array}{l}\text { History of thoracic surgery: } \\
\text { No }(n=69) \\
\text { Yes }(n=69)\end{array}$ & $\begin{array}{l}96.8 \\
96.9\end{array}$ & $\begin{array}{l}92.4- \\
100 \\
90.9- \\
100\end{array}$ & $\begin{array}{l}96.8 \\
93.2\end{array}$ & $\begin{array}{l}92.4- \\
100 \\
84.0- \\
100\end{array}$ & 0.944 \\
\hline $\begin{array}{l}\text { Reason of non-operability: } \\
\text { Surgery refused by patient }(n= \\
5) \\
\text { Surgically non operable }(n=27) \\
\text { Medically non operable }(n=70) \\
\text { Systemic disease }(n=36)\end{array}$ & $\begin{array}{c}100 \\
100 \\
98.6 \\
96.8\end{array}$ & $\begin{array}{l}81.4- \\
100 \\
\\
95.9- \\
100 \\
90.6- \\
100\end{array}$ & $\begin{array}{c}100 \\
77.1 \\
98.6 \\
96.8\end{array}$ & $\begin{array}{l}48.8- \\
100 \\
\\
95.9- \\
100 \\
90.6- \\
100\end{array}$ & 0.333 \\
\hline $\begin{array}{l}\text { Primary tumor }(n=74) \\
\text { Metastasis }(n=64)\end{array}$ & $\begin{array}{l}95.9 \\
98.2\end{array}$ & $\begin{array}{l}89.9- \\
100 \\
94.7- \\
100\end{array}$ & $\begin{array}{l}92.3 \\
98.2\end{array}$ & $\begin{array}{l}83.4- \\
100 \\
94.7- \\
100\end{array}$ & 0.416 \\
\hline
\end{tabular}

Missing values $(n=4)$ 


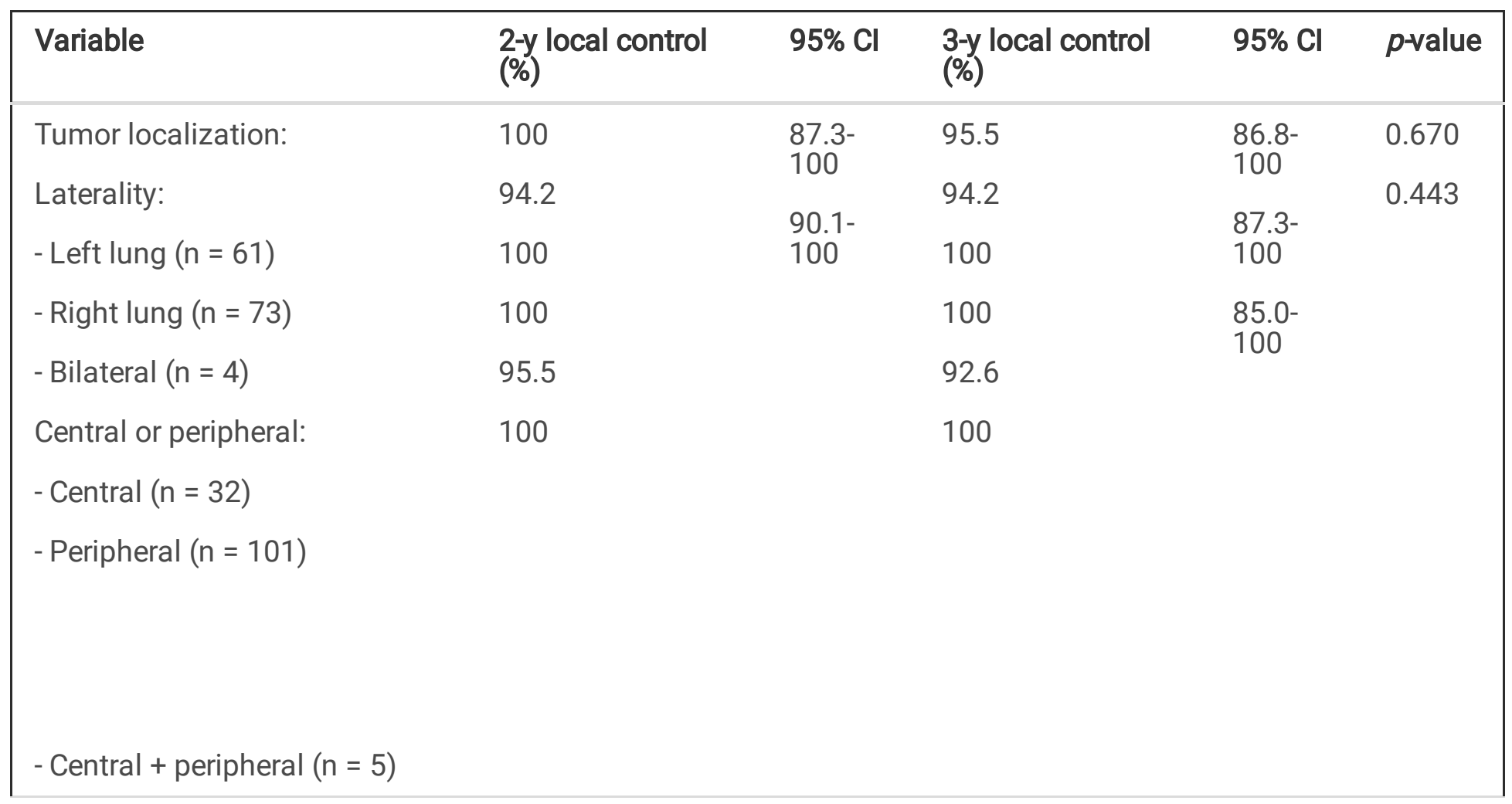

Missing values $(n=4)$ 


\begin{tabular}{|c|c|c|c|c|c|}
\hline Variable & $\begin{array}{l}\text { 2-y local control } \\
(\%)\end{array}$ & $95 \% \mathrm{Cl}$ & $\begin{array}{l}\text { 3-y local control } \\
(\%)\end{array}$ & $95 \% \mathrm{Cl}$ & $p$-value \\
\hline Histology: & 96.2 & \multirow{2}{*}{$\begin{array}{l}88.8- \\
100\end{array}$} & 96.2 & \multirow{2}{*}{$\begin{array}{l}88.8- \\
100\end{array}$} & 0.519 \\
\hline Histological proof: & 97.5 & & 93.7 & & 0.837 \\
\hline- no $(n=55)$ & 83.3 & $\begin{array}{l}94.0- \\
100\end{array}$ & 83.3 & $\begin{array}{l}85.8- \\
100\end{array}$ & \\
\hline -yes $(n=83)$ & 100 & \multirow{2}{*}{$\begin{array}{l}53.5- \\
100\end{array}$} & 95.0 & $\begin{array}{l}53.5- \\
100\end{array}$ & \\
\hline Histological type: & 96.6 & & 96.6 & & \\
\hline$-\operatorname{NSCLC}(n=5)$ & 100 & $\begin{array}{l}90.0- \\
100\end{array}$ & 100 & $\begin{array}{l}85.5- \\
100\end{array}$ & \\
\hline \multirow[t]{2}{*}{ - adenocarcinoma $(n=46)$} & \multirow[t]{2}{*}{100} & \multirow[t]{2}{*}{$\begin{array}{l}79.4- \\
100\end{array}$} & 100 & \multicolumn{2}{|l|}{$\begin{array}{l}90.0- \\
100\end{array}$} \\
\hline & & & & \multicolumn{2}{|l|}{$\begin{array}{l}79.4- \\
100\end{array}$} \\
\hline - squamous cell $(n=35)$ & 100 & & 100 & & \\
\hline \multirow{2}{*}{$\begin{array}{l}\text { - adenoid cystic carcinoma }(n= \\
4)\end{array}$} & 100 & & 100 & & \\
\hline & 100 & & 100 & & \\
\hline \multirow{2}{*}{$\begin{array}{l}\text { - melanoma }(n=5) \\
\text { - sarcoma }(n=2) \\
\text { - hepatocellular carcinoma }(n= \\
\text { 1) }\end{array}$} & 100 & & 100 & & \\
\hline & 92.9 & & 92.9 & & \\
\hline \multicolumn{6}{|l|}{ - mesothelioma $(n=1)$} \\
\hline \multicolumn{6}{|l|}{ - nerves $(n=1)$} \\
\hline \multicolumn{6}{|l|}{ - not known $(n=38)$} \\
\hline Number of metastatic sites: & 100 & $91.2-$ & 100 & $91.2-$ & 0.948 \\
\hline $0(n=1)$ & 97.0 & & 97.0 & & \\
\hline $1(\mathrm{n}=38)$ & 100 & & 100 & & \\
\hline \multicolumn{6}{|l|}{$\geqslant 2(n=26)$} \\
\hline PTV volume [cc]: & 98.0 & $94.0-$ & 93.7 & $84.7-$ & 0.904 \\
\hline$\leqslant 8.1(n=65)$ & 95.6 & & 95.6 & & \\
\hline$>8.1(\mathrm{n}=64)$ & & 100 & & 100 & \\
\hline
\end{tabular}

\section{Survival}

Median follow up was 28.7 months (22.7-35.6 months, $\mathrm{Cl} 95 \%$ ). Overall survival (OS) rates at 2 and 3 years were respectively $58.5 \%(\mathrm{Cl} 95 \%, 48.4-68.6 \%)$ and $35.7 \%$ (Cl 95\%, 25.2-45.9\%), with no difference regarding both SBRT modalities. Metastasis-free survival (MFS) and progression-free survival (PFS) significantly decreased in case of 
first recurrence at brain (13.5 vs 5.5 months, $p=0.007$ and 0.010 , respectively). MFS and PFS were significantly longer when treated lesions were primary lung tumors compared to metastases, while median MFS was not reached and PFS nearly doubled in case of primary lung cancer. However this last factor was not confirmed after multivariate analysis. Median survival, significantly decreased in case of first metastatic relapse occurred before 13.1 months. Median OS was 37.8 months when first metastatic relapse occurred before 13.1 months compared to a median OS of 64.5 months when first metastatic relapse occurred after 13.1 months $(p=0.035)$, and remained predictive after Cox-regression $(p<0.0001)$. Other predictive factors for survival $(O S, M F S$, and PFS) are depicted in Table 3. 
Table 3

Predictive factors for survival (OS, MFS, and PFS)

\begin{tabular}{|c|c|c|c|c|c|c|}
\hline Variable & $\begin{array}{l}\text { Median } \\
\text { overall } \\
\text { survival } \\
\text { (OS) } \\
\text { [months] }\end{array}$ & $\begin{array}{l}\text { p-value } \\
\text { (log- } \\
\text { rank/Cox } \\
\text { regression) }\end{array}$ & $\begin{array}{l}\text { Median } \\
\text { metastasis- } \\
\text { free } \\
\text { survival } \\
\text { (MFS) } \\
\text { [months] }\end{array}$ & $\begin{array}{l}\text { p-value(log- } \\
\text { rank/Cox } \\
\text { regression) }\end{array}$ & $\begin{array}{l}\text { Median } \\
\text { progression- } \\
\text { free survival } \\
\text { (PFS) } \\
\text { [months] }\end{array}$ & $\begin{array}{l}\text { p-value(log- } \\
\text { rank/Cox } \\
\text { regression) }\end{array}$ \\
\hline $\begin{array}{l}\text { Age [years]: } \\
\leq 71(n=54) \\
>71(n=57)\end{array}$ & $\begin{array}{l}64.5 \\
40.1\end{array}$ & 0.945 & $\begin{array}{l}25.9 \\
\text { NR }\end{array}$ & $0.037 * / N S$ & $\begin{array}{l}24.8 \\
\text { NR }\end{array}$ & $0.075 / 0.02$ \\
\hline $\begin{array}{l}\text { Radiotherapy } \\
\text { technique: } \\
\text { CyberKnife }(n= \\
71) \\
\text { Other }(n=40)\end{array}$ & $\begin{array}{l}\text { NR } \\
64.5\end{array}$ & 0.338 & $\begin{array}{l}27.9 \\
48.0\end{array}$ & 0.334 & $\begin{array}{l}25.9 \\
48.0\end{array}$ & 0.179 \\
\hline $\begin{array}{l}\text { Smoker: } \\
\text { No }(n=39) \\
\text { Yes }(n=72)\end{array}$ & $\begin{array}{l}41.1 \\
64.5\end{array}$ & 0.459 & $\begin{array}{l}25.9 \\
48.0\end{array}$ & 0.976 & $\begin{array}{l}25.9 \\
28.8\end{array}$ & 0.941 \\
\hline $\begin{array}{l}\text { History of } \\
\text { thoracic } \\
\text { irradiation: } \\
\text { No }(n=84) \\
\text { Yes }(n=27)\end{array}$ & $\begin{array}{l}64.5 \\
29.6\end{array}$ & $0.192 / N S$ & $\begin{array}{l}45.0 \\
28.4\end{array}$ & 0.826 & $\begin{array}{l}68.9 \\
48.7\end{array}$ & 0.76 \\
\hline $\begin{array}{l}\text { Reason of non- } \\
\text { operability: } \\
\text { Surgery refused } \\
(n=5)\end{array}$ & $\begin{array}{l}\text { NR } \\
40.1 \\
\text { NR }\end{array}$ & 0.820 & $\begin{array}{l}\text { NR } \\
45.0 \\
48.0\end{array}$ & $0.114 / \mathrm{NS}$ & $\begin{array}{l}\text { NR } \\
28.8 \\
48\end{array}$ & $0.15 / \mathrm{NS}$ \\
\hline $\begin{array}{l}\text { Surgically inop } \\
(n=24) \\
\text { Medically inop } \\
(n=49) \\
\text { Systemicdisease } \\
(n=33)\end{array}$ & 41.1 & & 19.3 & & 17.7 & \\
\hline
\end{tabular}

$\mathrm{NR}=$ not reached

NS : not significant

inop :inoperable 


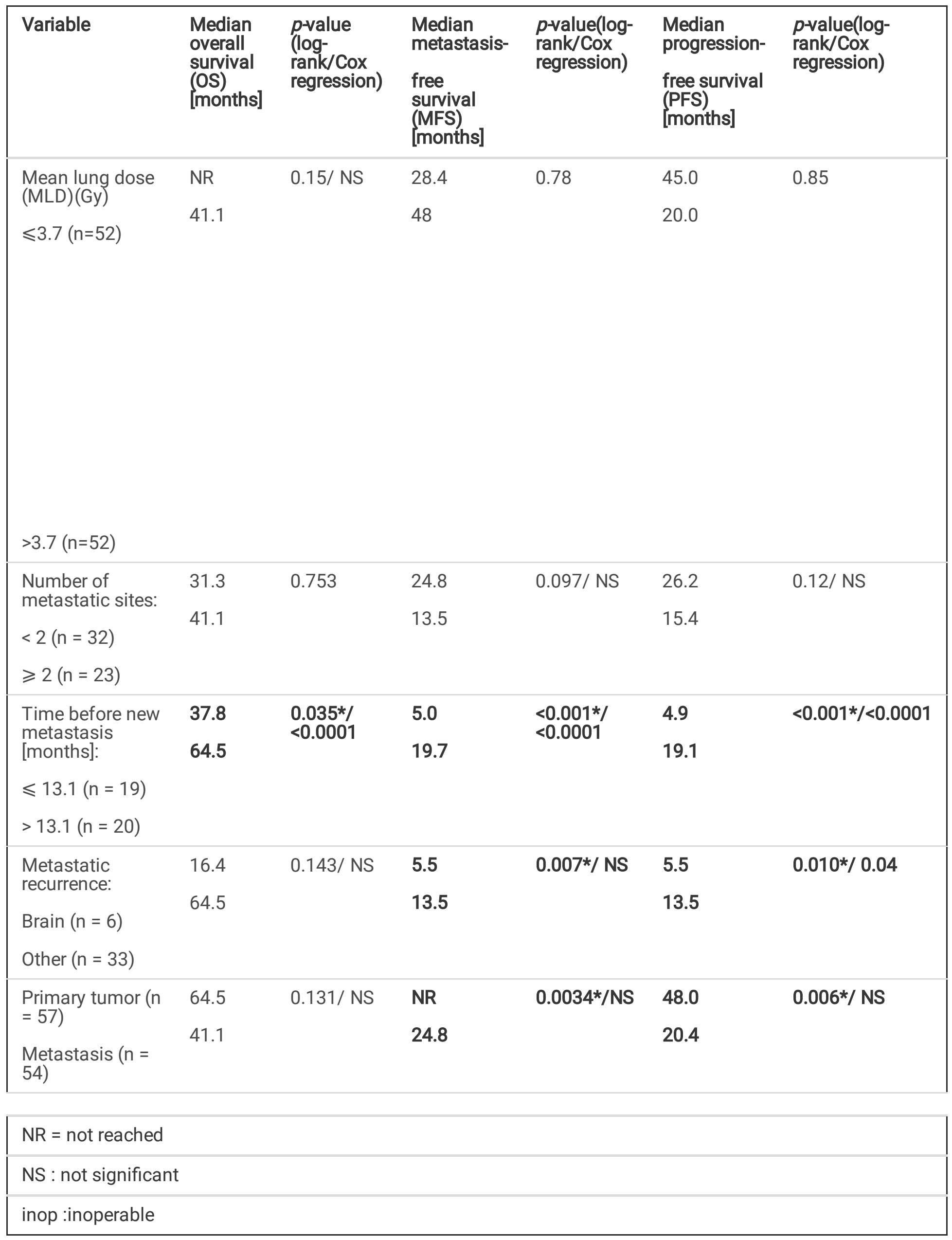




\begin{tabular}{|c|c|c|c|c|c|c|}
\hline Variable & $\begin{array}{l}\text { Median } \\
\text { overall } \\
\text { survival } \\
\text { (OS) } \\
\text { [months] }\end{array}$ & $\begin{array}{l}\text { p-value } \\
\text { (log- } \\
\text { rank/Cox } \\
\text { regression) }\end{array}$ & $\begin{array}{l}\text { Median } \\
\text { metastasis- } \\
\text { free } \\
\text { survival } \\
\text { (MFS) } \\
\text { [months] }\end{array}$ & $\begin{array}{l}p \text {-value(log- } \\
\text { rank/Cox } \\
\text { regression) }\end{array}$ & $\begin{array}{l}\text { Median } \\
\text { progression- } \\
\text { free survival } \\
\text { (PFS) } \\
\text { [months] }\end{array}$ & $\begin{array}{l}p \text {-value(log- } \\
\text { rank/Cox } \\
\text { regression) }\end{array}$ \\
\hline V5\% [cc]: & 64.5 & 0.779 & 45.0 & 0.91 & 27.0 & 0.589 \\
\hline$\leqslant 17.2(n=52)$ & 41.1 & 27.8 & 27.9 & 0.291 & 27.9 & 0.64 \\
\hline$>17.2(n=52)$ & NR & 0.28 & 24.8 & & 25.7 & \\
\hline $\begin{array}{l}\text { Composite V5\% } \\
\text { [cc]: }\end{array}$ & 29.57 & & 28.4 & & NR & \\
\hline \multicolumn{7}{|l|}{$\leqslant 37(n=9)$} \\
\hline \multicolumn{7}{|l|}{$>37(n=10)$} \\
\hline PTV volume [cc]: & NR & 0.722 & 27.9 & 0.222 & 27.0 & 0.300 \\
\hline$\leqslant 8.1(n=52)$ & 41.1 & & 48.0 & & 48.0 & \\
\hline \multicolumn{7}{|l|}{$>8.1(n=52)$} \\
\hline \multicolumn{7}{|l|}{$\mathrm{NR}=$ not reached } \\
\hline \multicolumn{7}{|l|}{ NS : not significant } \\
\hline inop :inoperable & & & & & & \\
\hline
\end{tabular}

\begin{tabular}{|llll|}
\hline Variable & Relative Risk & SD & p-value \\
\hline OS & & & \\
\hline Time to first metastasis $<13.1$ months & 9.0 & 0.02 & $<0.0001$ \\
\hline PFS & & & \\
\hline Age $>71$ years & 1.2 & 0.37 & 0.02 \\
\hline Time to first metastasis $<13.1$ months & 2.6 & 0.06 & $<0.0001$ \\
\hline Metastatic recurrence at brain & 1.2 & 0.6 & 0.04 \\
\hline
\end{tabular}

\section{Toxicity}

Patients presented at least one toxicity in $68.5 \%$ of the cases, mainly grade 1 acute toxicity $(n=50)$. About three quarter of patients did not develop any late toxicity (73.9\%), 3 patients presented a late grade 3 toxicity (Table 1 ). Toxicity incidence significantly increased beyond the median V5 value of $17.2 \%(56.0$ vs $77.4 \%, p=0.021)$. Toxicity is described in Table 4. 
Table 4

Predicitive factors for toxicity

\begin{tabular}{|c|c|c|c|c|c|c|c|c|c|}
\hline \multirow[t]{2}{*}{ Variable } & \multicolumn{2}{|c|}{ Toxicity (\%) } & \multirow[t]{2}{*}{$\begin{array}{l}p- \\
\text { value }\end{array}$} & \multicolumn{2}{|c|}{$\begin{array}{l}\text { Acute toxicity } \\
\text { (\%) }\end{array}$} & \multirow[t]{2}{*}{$\begin{array}{l}p- \\
\text { value }\end{array}$} & \multicolumn{2}{|c|}{$\begin{array}{l}\text { Late toxicity } \\
\text { (\%) }\end{array}$} & \multirow[t]{2}{*}{$\begin{array}{l}p- \\
\text { value }\end{array}$} \\
\hline & Yes & No & & Yes & No & & Yes & No & \\
\hline CK $(n=71)$ & 70.4 & 29.6 & 0.557 & 54.9 & 45.1 & 0.793 & 28.2 & 71.8 & 0.511 \\
\hline Others $(n=40)$ & 65.0 & 35.0 & & 57.5 & 42.5 & & 22.5 & 77.5 & \\
\hline PTV volume [cc]: & 65.4 & 34.6 & 0.486 & 50.0 & 50.0 & 0.205 & 21.2 & 78.8 & 0.288 \\
\hline$\leqslant 8.1(n=52)$ & 71.7 & 28.3 & & 62.3 & 37.7 & & 30.2 & 69.8 & \\
\hline \multicolumn{10}{|l|}{$>8.1(n=52)$} \\
\hline V5\% [cc]: & 56.0 & 44.0 & $0.021 *$ & 46.0 & 54.0 & 0.063 & 22.0 & 78.0 & 0.601 \\
\hline$\leqslant 17.2(n=52)$ & 77.4 & 22.6 & & 64.2 & 35.8 & & 26.4 & 73.6 & \\
\hline \multicolumn{10}{|l|}{$>17.2(n=52)$} \\
\hline V20\% [cc]: & 60.4 & 39.6 & 0.140 & 50.9 & 49.1 & 0.355 & 18.9 & 81.1 & 0.187 \\
\hline$<2.4(\mathrm{n}=52)$ & 74.0 & 26.0 & & 60.0 & 40.0 & & 30.0 & 70.0 & \\
\hline \multicolumn{10}{|l|}{$>2.4(n=52)$} \\
\hline Meanlung dose (MLD) & 61.5 & 38.5 & 0.209 & 50.0 & 50.0 & 0.236 & 25.0 & 75.0 & 1.000 \\
\hline$<3.7(n=52)$ & 73.1 & 26.9 & & 61.5 & 38.5 & & 25.0 & 75.0 & \\
\hline \multicolumn{10}{|l|}{$>3.7(n=52)$} \\
\hline Cumulative MLD [Gy]: & 61.1 & 38.9 & 0.141 & 53.7 & 46.3 & 0.597 & 22.2 & 77.8 & 0.535 \\
\hline$<7.9(\mathrm{n}=12)$ & 74.5 & 25.5 & & 58.8 & 41.2 & & 27.5 & 72.5 & \\
\hline$>7.9(\mathrm{n}=8)$ & & & & & & & & & \\
\hline
\end{tabular}

Table 5. CyberKnife (CK) versus other techniques: outcome, dosimetry, and toxicity 


\section{Local control}

\begin{tabular}{|c|c|c|c|c|c|c|c|}
\hline \multicolumn{2}{|l|}{ Variable } & \multicolumn{2}{|c|}{$\begin{array}{l}\text { 2-year local control } \\
\text { (\%) }\end{array}$} & $95 \% \mathrm{Cl}$ & $\begin{array}{l}\text { 3-year } \\
\text { local } \\
\text { control } \\
\text { (\%) }\end{array}$ & $95 \% \mathrm{Cl}$ & $p$-value \\
\hline \multicolumn{2}{|c|}{ CyberKnife $(n=82)$} & \multicolumn{2}{|l|}{96.2} & $90.8-100$ & 93.3 & $85.6-100$ & \multirow[t]{2}{*}{0.68} \\
\hline Other $(n=$ & & 98.2 & & $94.4-100$ & 98.2 & $94.4-100$ & \\
\hline \multicolumn{8}{|l|}{ Survival } \\
\hline Variable & \multicolumn{2}{|c|}{$\begin{array}{l}\text { Medianoverallsurvival } \\
\text { [months] }\end{array}$} & $\mathrm{p}$-value & $\begin{array}{l}\text { Median } \\
\text { metastasis-free } \\
\text { survival (MFS) } \\
\text { [months] }\end{array}$ & $\mathrm{p}$-value & $\begin{array}{l}\text { Median } \\
\text { progression-free } \\
\text { survival (PFS) } \\
\text { [months] }\end{array}$ & $\mathrm{p}$-value \\
\hline $\begin{array}{l}\mathrm{CK}(\mathrm{n}= \\
71)\end{array}$ & \multicolumn{2}{|l|}{ NR } & 0.338 & 27.9 & 0.334 & 25.9 & \multirow[t]{2}{*}{0.18} \\
\hline $\begin{array}{l}\text { Others(n } \\
=40)\end{array}$ & \multicolumn{3}{|l|}{64.5} & \multicolumn{2}{|l|}{48.0} & 48.0 & \\
\hline
\end{tabular}

\section{Dosimetry characteristics for CyberKnife (CK) versus VMAT treatments}

\begin{tabular}{|c|c|c|c|}
\hline Variable & Mean for CK & Mean for VMAT & $p$-value \\
\hline \multirow[t]{2}{*}{ GTV volume [cc]: } & $(n=81)$ & $(n=52)$ & 0.84 \\
\hline & 2.1 & 1.4 & \\
\hline \multirow[t]{2}{*}{ PTV volume [cc]: } & $(n=81)$ & $(n=52)$ & $0.013^{*}$ \\
\hline & 12.3 & 21.9 & \\
\hline \multirow[t]{2}{*}{ Ratio GTV/reduced PTV in CK } & $(n=81)$ & $(n=52)$ & $0.0013^{*}$ \\
\hline & 2.9 & 4.8 & \\
\hline \multirow[t]{2}{*}{ Meanlung dose (MLD) [Gy]: } & $(n=79)$ & $(n=51)$ & $<0.0001 *$ \\
\hline & 2.6 & 4.1 & \\
\hline \multirow[t]{2}{*}{ Volume of lung receiving $5 \mathrm{~Gy}$ (V5\%) [cc]: } & $(n=79)$ & $(n=51)$ & $0.002 *$ \\
\hline & 13.5 & 19.9 & \\
\hline \multirow[t]{2}{*}{ Volume of lung receiving 20Gy (V20\%) [cc]: } & $(n=79)$ & $(n=51)$ & $<0.0001 *$ \\
\hline & 2.3 & 5.4 & \\
\hline \multirow[t]{2}{*}{ Composite MLD [Gy]: } & $(n=19)$ & $(n=19)$ & 0.849 \\
\hline & 7.8 & 7.5 & \\
\hline \multirow[t]{2}{*}{ Composite V5\% [cc]: } & $(n=19)$ & $(n=19)$ & 0.513 \\
\hline & 40.4 & 35.9 & \\
\hline
\end{tabular}




\begin{tabular}{|llll}
\hline Local control & & & \\
\hline Variable & Proportion for CK (\%) & Proportion for VMAT (\%) & p-value \\
\hline Toxicity: & $\mathbf{n}=\mathbf{7 1}$ & 35.0 & 0.56 \\
No & 70.6 & 65.0 & \\
Yes & & & 0.79 \\
Acute toxicity: & 45.1 & 42.5 & 0.98 \\
No & & & \\
Yes & 54.9 & 57.5 & \\
Grade 0 & 43.7 & 42.5 & \\
Grade 1 & 45.1 & 45.0 & 0.51 \\
Grade 2 & 9.9 & 10.0 & 0.26 \\
Grade 3 & 1.3 & 2.5 & \\
Late toxicity: & 71.8 & 77.5 & \\
No & 28.2 & 22.5 & \\
Yes & 71.8 & 77.5 & \\
Grade 0 & 15.5 & 5.0 & \\
Grade 1 & 11.3 & 12.5 & \\
Grade 2 & 1.4 & 5.0 & \\
Grade 3 & & & \\
\hline CK & & & \\
\hline
\end{tabular}

\section{CK versus other treatment modalities}

Local control rate were comparable between CK and other treatment modalities, with a local control rate of $96.2 \%$ vs $98.2 \%$, at 2 years and $93.3 \%$ vs $98.2 \%$ at 3 years, $p=0.68$ (Figure1). OS, MFS, and PFS were not influenced by the choice of the machine neither.

Dosimetric analysis revealed significant differences depending of the choice of the machine. GTV were comparable between $\mathrm{CK}$ and other modalities ( $2.1 \mathrm{vs} 1.4 \mathrm{cc}, \mathrm{p}=0.84)$. Nevertheless, treatments with $\mathrm{CK}$ resulted in significantly smaller PTV (12.3 vs $21.9 \mathrm{cc}, p=0.013)$, and were associated with significant dosimetric benefit with lower MLD ( 2.6 vs $4.1 \mathrm{~Gy}, \mathrm{p}<0.001)$ ), lower V5 (13.5 vs $19.9 \%, \mathrm{p}=0.002)$ and lower V20 (means at 2.3 vs $5.4 \%, p<0.001)$. This dosimetric benefit was not associated with a clear clinical benefit after SBRT. There was no significant difference in terms of incidence of toxicity $(p=0.56)$, in acute toxicity $(p=0.79)$, in late toxicity $(p=$ $0.51)$, or in terms of maximal grade of toxicity acute and late toxicity $(p=0.98$ and $p=0.26)$.

\section{Discussion}


This retrospective study describes factors associated with outcome, toxicity, and dosimetric parameters after lung SBRT, depending on the chosen delivery device. Our results revealed excellent local control rates of $96.8 \%$ and $94.8 \%$ at 2 and 3 years after SBRT, and a low rate of moderate-severe toxicity, values that are comparable with the literature(11-13). We have not obtained a significant difference in terms of local control rate after SBRT depending on the technique choice.

Claude et al, prospectively analyzed the efficiency and toxicity of SBRT with CK compared to linear accelerator SBRT treatments for peripheral early stage NSCLC. A total of 68 patients were treated using a linac and 38 by means of CK. Authors have not described a significant difference in terms of local control depending on the employed device; 2-year local control rate was 97.0\% (95\% Cl: 90.6-99.4\%) with linear accelerator vs 100\% with CK, respectively $(p=0.2839)$. Neither, acute and late toxicity differed between the 2 modalities. However, we can notice that this study did not report the dosimetrical parameters specific to the device choice. The study of Claude et al, represents the only prospective trial other that confronted the two modalities; however, we can underline that the treatments were not performed in the same institution (multicentric study), and could induce bias for this direct comparison(13). Even retrospective, our study represents the first one directly comparing the outcomes of patients receiving lung SBRT using three different modalities in the same radiotherapy department.

In our study, all patients that presented a local relapse were older than 71 years old. We identified that oldest patients presented a significantly lower local control compared to younger ones. A retrospective study of 219 patients also reported that age significantly influenced the local outcome after SBRT(14). On the other hand, Watanabe, et al depicted a preserved local control in a cohort of 64 patients older than 80 years old, which was $98.4 \%$ at 3 years $(15)$.

In our cohort about $40 \%$ of patients were treated without histological proof of malignancy and indication for irradiation was based on clinical and radiological criterias and this subset of patients presented similar outcomes compared to those with a histological proof of malignancy. In this context, several publications already showed comparable outcomes in patients after empiric SBRT, supporting the hypothesis that patients did have lung cancers(16-18).

Loco-regional control was good and comparable to other studies of lung SBRT and also in compare to surgical outcomes after video-assisted thoracoscopic surgical lobectomy with mediastinal lymph node dissection(19).

OS was significantly decreased in case of early metastatic recurrence (< 13.1 months), and PFS was significantly shorter when the first identified relapse site was the brain corresponding to the usual clinical factors and influencing the outcome in metastatic patients $(20,21)$.

More than half of our patients presented at least one toxicity (68.5\%), and the most common form was Grade 1 acute toxicity (79.3\%). About three quarter (73.9\%) of patients have not developed any late toxicity. Incidence of toxicity was comparable with the literature(22-25). In our cohort, several dosimetric parameters were analyzed in order to identify predictive factors for toxicity such as PTV, V5, V20, MLD, and composite MLD. Compared to other techniques, and even though comparable GTV, CK treatments significantly permitted to decrease PTV, MLD, V5, and V20; which are dosimetric parameters usually found to be related with toxicity $(26,27)$. In our cohort, an increased V5 value correlated with a higher incidence of toxicity. Reducing the V 5 value could be particularly interesting in case of multiple courses of SBRT lung irradiations(27). Hence, even if we not found a significant difference regarding toxicity in relation to the delivery device employed, it can be appropriate to favor CK

Page 22/28 
irradiation in order to improve dosimetric parameters. Indeed, our study showed that cumulative MLD and cumulative V5 values are approximately doubled in case of multiple courses of lung irradiations, and techniques able to spare healthy lung reducing these dosimetric parameters could be preferred from the first lung SBRT. Moreover, CK could be particularly of interest in the event of local relapse re-irradiation, situation known to be at risk of high rates of severe toxicity $(28,29)$.

\section{Conclusion}

Compared to other SBRT techniques, CK treatments are able to treat comparable GTV with reduced PTV, MLD, V5, and V20. The dosimetric benefit of CK irradiation was not associated with a clinical benefit, with comparable outcome in terms of control rates and toxicity. Toxicity incidence was less frequent when reducing V5. Altogether, it seems appropriate to favor the use of CK, particularly in the event of multiple courses of irradiations or in case of re-irradiation.

\section{Abbreviations}

SBRT

stereotactic body radiotherapy

NSCLC

non-small-cell lung cancer

CHUV

University Hospital Center of Lausanne (Centre Hospitalier Universitaire Vaudois)

VMAT

volumetric modulated arc therapy

Linac

linear accelerator

CT

computed tomography

$\mathrm{ABC}$

Active Breath Control ${ }^{\circledR}$

CK

CyberKnife ${ }^{\circledR}$

GTV

Gross tumor volume

ITV

Internal target volume

CTV

Clinical target volume

PTV

Planning target volume

WHO

World Health Organization

MLD

mean lung dose 
V5

Percent volume of lung receiving 5Gy

V20

Percent volume of lung receiving 20Gy

OS

overall survival

$\mathrm{Cl}$

confidence interval

MFS

metastasis-free survival

PFS

progression-free survival

LC

local control

\section{Declarations}

\section{Ethics approval and consent to participate}

This project met the regulatory requirements of the Swiss Federal Law on Research on Human Beings (LRH) and the Swiss Ordinance on Research on Human Being (ORH). The study protocol obtained the prior approval of the Swiss cantonal commission for the ethics of research on human beings (CER-VD).

Registered on February 24th 2021, ID 2021-00267, with the authorization of the CER-VD ethics committee (Switzerland).

\section{Consent for publication}

Not applicable for that section.

\section{Availability of data and materials}

The datasets used and analysed during the current study are available from the corresponding author on reasonable request.

\section{Competing interests}

The authors declare that they have no competing interests.

\section{Funding}

No specific funding was received for this investigation. The investigators have no conflict of interest in this project.

\section{Authors' contributions}


ELR, EMO, and RK collected and analyzed the patient data, and were major contributors in writing the manuscript. $A C, A D, H B, E R O, A L, C V G$ and TK contributed in writing the manuscript. All authors read and approved the final manuscript.

\section{Acknowledgements}

No.

\section{References}

1. Fitzmaurice $C$, Abate $D$, Abbasi N, et al. Global, regional, and national cancer incidence, mortality, years of life lost, years lived with disability, and disability-Adjusted life-years for 29 cancer groups, 1990 to 2017: A systematic analysis for the global burden of disease study. JAMA Oncol. 2019;5:1749-68. https://doi.org/10.1001/jamaoncol.2019.2996.

2. Palma D, Visser O, Lagerwaard FJ, Belderbos J, Slotman BJ, Senan S. Impact of introducing stereotactic lung radiotherapy for elderly patients with stage I non-small-cell lung cancer: A population-based time-trend analysis. J Clin Oncol. 2010;28:5153-9. https://doi.org/10.1200/JC0.2010.30.0731.

3. Haasbeek CJA, Palma D, Visser O, Lagerwaard FJ, Slotman B, Senan S. Early-stage lung cancer in elderly patients: A population-based study of changes in treatment patterns and survival in the Netherlands. Ann Oncol. 2012;23:2743-7. https://doi.org/10.1093/annonc/mds081.

4. Weder W, Moghanaki D, Stiles B, Siva S, Rocco G. The great debate flashes: Surgery versus stereotactic body radiotherapy as the primary treatment of early-stage lung cancer. Eur J Cardio-thoracic Surg. 2018;53:295305. https://doi.org/10.1093/ejcts/ezx410.

5. Guckenberger M, Andratschke N, Dieckmann K, et al. ESTRO ACROP consensus guideline on implementation and practice of stereotactic body radiotherapy for peripherally located early stage non-small cell lung cancer. Radiother Oncol. 2017;124:11-7. https://doi.org/10.1016/j.radonc.2017.05.012.

6. Videtic GMM, Woody NM, Reddy CA, Stephans KL. Never too old: A single-institution experience of stereotactic body radiation therapy for patients 90 years and older with early stage lung cancer. Pract Radiat Oncol. 2017;7:e543-9. https://doi.org/10.1016/j.prro.2017.06.011.

7. Postmus PE, Kerr KM, Oudkerk M, et al. Early and locally advanced non-small-cell lung cancer (NSCLC): ESMO Clinical Practice Guidelines for diagnosis, treatment and follow-up. Ann Oncol. 2017;28:iv1-21. https://doi.org/10.1093/annonc/mdx222.

8. Palma DA, Olson R, Harrow S, et al. Stereotactic ablative radiotherapy versus standard of care palliative treatment in patients with oligometastatic cancers (SABR-COMET): a randomised, phase 2, open-label trial. Lancet. 2019;393:2051-8. https://doi.org/10.1016/S0140-6736(18)32487-5.

9. Bondiau PY, Bénézery K, Beckendorf V, et al. Radiothérapie stéréotaxique robotisée par CyberKnife ${ }^{\circ}$ : aspects techniques et indications. Cancer/Radiotherapie. 2007;11:338-44.

https://doi.org/10.1016/j.canrad.2007.09.146.

10. Kilby W, Dooley JR, Kuduvalli G, Sayeh S, Maurer CR. The CyberKnife® robotic radiosurgery system in 2010. Technol Cancer Res Treat. 2010;9:433-52. https://doi.org/10.1177/153303461000900502.

11. Gutiérrez E, Sánchez I, Díaz O, et al. Current evidence for stereotactic body radiotherapy in lung metastases. Curr Oncol. 2021;28:2560-78. https://doi.org/10.3390/curroncol28040233. 
12. Virbel G, Fèvre C, Le, Noël G, Antoni D. Stereotactic body radiotherapy for patients with lung oligometastatic disease: A five-year review. Cancers (Basel) 2021;13. https://doi.org/10.3390/cancers13143623.

13. Claude L, Morelle M, Mahé MA, et al. A comparison of two modalities of stereotactic body radiation therapy for peripheral early-stage non-small cell lung cancer: Results of a prospective French study. Br J Radiol 2020;93. https://doi.org/10.1259/bjr.20200256.

14. Kessel KA, Grosser RCE, Kraus KM, Hoffmann H, Oechsner M, Combs SE. Stereotactic body radiotherapy (SBRT) in patients with lung metastases-prognostic factors and long-term survival using patient self-reported outcome (PRO). BMC Cancer 2020;20. https://doi.org/10.1186/s12885-020-6635-8.

15. Watanabe K, Katsui K, Sugiyama S, et al. Lung stereotactic body radiation therapy for elderly patients aged $\geq$ 80 years with pathologically proven early-stage non-small cell lung cancer: a retrospective cohort study. Radiat Oncol 2021;16. https://doi.org/10.1186/s13014-021-01769-7.

16. Berman AT, Jabbour SK, Vachani A, et al. Empiric radiotherapy for lung cancer collaborative group multiinstitutional evidence-based guidelines for the use of empiric stereotactic body radiation therapy for nonsmall cell lung cancer without pathologic confirmation. Transl Lung Cancer Res. 2018;8:5-14. https://doi.org/10.21037/tlcr.2018.12.12.

17. Takeda A, Kunieda E, Sanuki N, Aoki Y, Oku Y, Handa H. Stereotactic body radiotherapy (SBRT) for solitary pulmonary nodules clinically diagnosed as lung cancer with no pathological confirmation: Comparison with non-small-cell lung cancer. Lung Cancer. 2012;77:77-82. https://doi.org/10.1016/j.lungcan.2012.01.006.

18. Verstegen NE, Lagerwaard FJ, Haasbeek CJA, Slotman BJ, Senan S. Outcomes of stereotactic ablative radiotherapy following a clinical diagnosis of stage i NSCLC: Comparison with a contemporaneous cohort with pathologically proven disease. Radiother Oncol. 2011;101:250-4. https://doi.org/10.1016/j.radonc.2011.09.017.

19. Chang JY, Mehran RJ, Feng L, et al. Stereotactic ablative radiotherapy for operable stage I non-small-cell lung cancer (revised STARS): Iong-term results of a single-arm, prospective trial with prespecified comparison to surgery. Lancet Oncol. 2021;22:1448-57. https://doi.org/10.1016/S1470-2045(21)00401-0.

20. Brundage MD, Davies D, Mackillop WJ. Prognostic Factors in Non-Small Cell Lung Cancer*A Decade of Progress.

21. Ren C, Ji T, Liu T, Dang J, Li G. The risk and predictors for severe radiation pneumonitis in lung cancer patients treated with thoracic reirradiation. Radiat Oncol 2018;13. https://doi.org/10.1186/s13014-018-1016Z.

22. Zhao J, Yorke ED, Li L, et al. Simple Factors Associated With Radiation-Induced Lung Toxicity After Stereotactic Body Radiation Therapy of the Thorax: A Pooled Analysis of 88 Studies. In: International Journal of Radiation Oncology Biology Physics. Vol 95. Elsevier Inc.; 2016. p. 1357-1366. https://doi.org/10.1016/j.ijrobp.2016.03.024.

23. Badra EV, Baumgartl M, Fabiano S, Jongen A, Guckenberger M. Stereotactic radiotherapy for early stage nonsmall cell lung cancer: Current standards and ongoing research. Transl Lung Cancer Res. 2021;10:1930-49. https://doi.org/10.21037/tlcr-20-860.

24. Dupic G, Biau J, Molnar I, et al. Significant Correlation Between Overall Survival and Mean Lung Dose in Lung Stereotactic Body Radiation Therapy (SBRT). Front Oncol 2020;10. https://doi.org/10.3389/fonc.2020.01577.

25. Matsuo Y, Shibuya K, Nakamura M, et al. Dose-volume metrics associated with radiation pneumonitis after stereotactic body radiation therapy for lung cancer. Int J Radiat Oncol Biol Phys 2012;83.

Page 26/28 
https://doi.org/10.1016/j.ijrobp.2012.01.018.

26. Saha A, Beasley M, Hatton N, et al. Clinical and dosimetric predictors of radiation pneumonitis in early-stage lung cancer treated with Stereotactic Ablative radiotherapy (SABR) - An analysis of UK's largest cohort of lung SABR patients. Radiother Oncol. 2021;156:153-9. https://doi.org/10.1016/j.radonc.2020.12.015.

27. Muller DA, Dutta SW, Aliotta E, et al. Clinical Outcomes and Predictors of Lung Toxicity After Multiple Courses of Lung Stereotactic Body Radiotherapy for Early-Stage Non-Small Cell Lung Cancer. Clin Lung Cancer. 2021;22:234-41. https://doi.org/10.1016/j.cllc.2020.06.006.

28. Kinj R, Casutt A, Bennassi A, et al. Stereotactic lung reirradiation for local relapse: A case series. Clin Transl Radiat Oncol. 2021;28:85-7. https://doi.org/10.1016/j.ctro.2021.03.007.

29. Kennedy WR, Gabani P, Nikitas J, Robinson CG, Bradley JD, Roach MC. Repeat stereotactic body radiation therapy (SBRT) for salvage of isolated local recurrence after definitive lung SBRT. Radiother Oncol. 2020;142:230-5. https://doi.org/10.1016/j.radonc.2019.08.010.

\section{Figures}

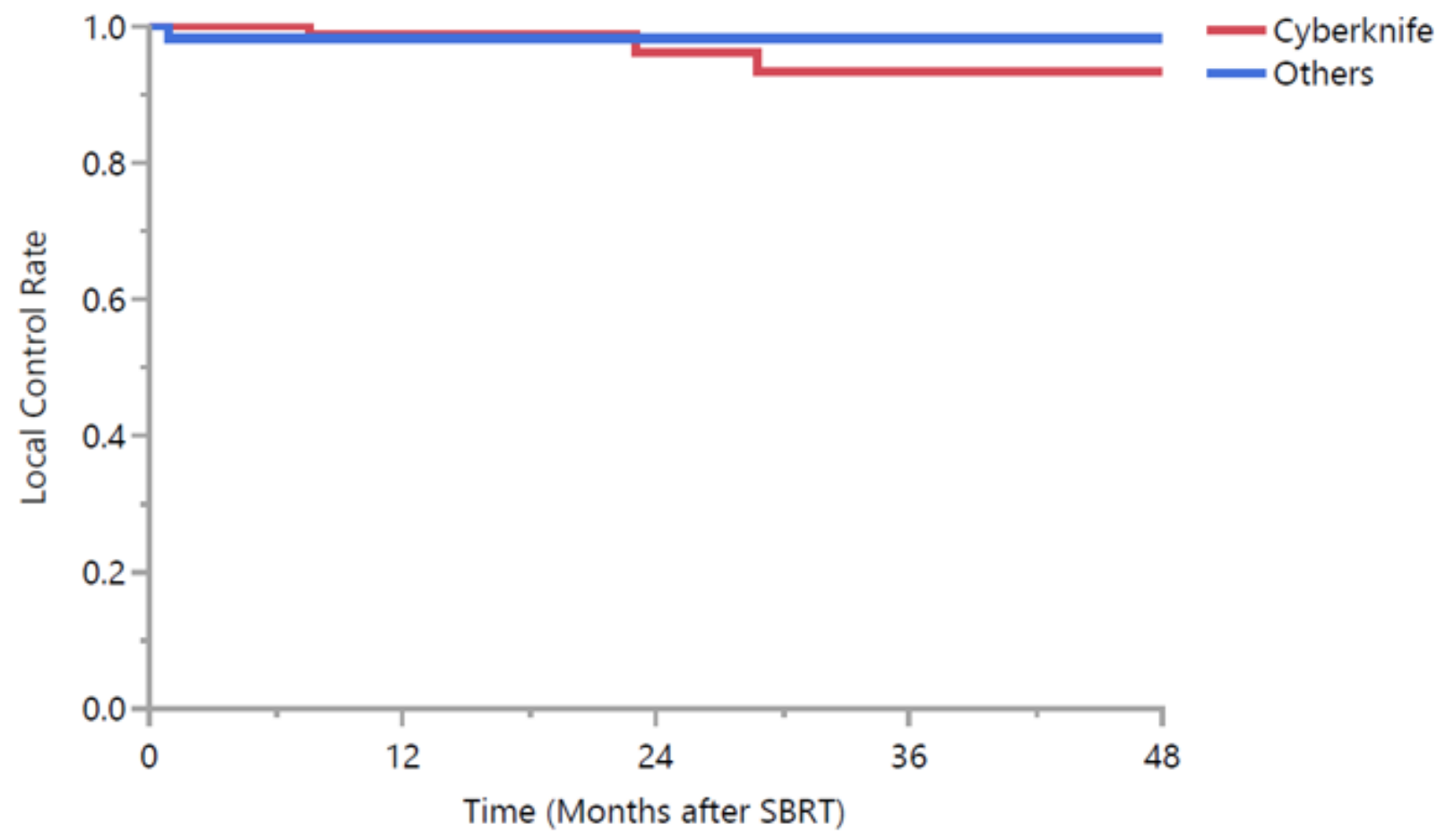

\begin{tabular}{|l|l|l|l|l|l|}
\hline $\begin{array}{l}\text { Number at risk } \\
\text { (cumulative } \\
\text { number of } \\
\text { events) }\end{array}$ & 0 months & 12 months & 24 months & 36 months & 48 months \\
\hline Cyberknife & $82(0)$ & $60(1)$ & $40(2)$ & $20(3)$ & $4(3)$ \\
\hline Others & $56(0)$ & $29(1)$ & $21(1)$ & $12(1)$ & $8(1)$ \\
\hline
\end{tabular}


Local control rate depending on the machine choice

Page 28/28 